\title{
Effects of the SGLT-2 Inhibitor Empagliflozin on Renal Tissue Oxygenation in Non-Diabetic Subjects: A Randomized, Double-Blind, Placebo-Controlled Study Protocol
}

\author{
Marie-Eve Muller · Menno Pruijm • Olivier Bonny • Michel Burnier • \\ Anne Zanchi
}

Received: February 22, 2018 / Published online: May 25, 2018

(C) The Author(s) 2018

\section{ABSTRACT}

Introduction: Empagliflozin is an SGLT-2 inhibitor (SGLT-2i) which belongs to a new class of hypoglycemic drugs with the unique property of decreasing blood glucose independently from insulin, through an increase in glycosuria. In addition to decreasing cardiovascular morbidity and mortality, empagliflozin has nephroprotective properties in high cardiovascular risk patients with type 2 diabetes. Decreased hyperfiltration and shifting towards more favorable renal fuel energetics with improved renal oxygenation may explain some of these properties. With this study, we propose to explore the effects of empagliflozin on renal tissue oxygenation using blood oxygenation level-dependent magnetic resonance imaging (BOLD-MRI). Methods: This is a double-blind, randomized, placebo-controlled study examining the acute

Enhanced digital features To view enhanced digital features for this article go to https://doi.org/10.6084/ m9.figshare.6189185.

M.-E. Muller · M. Pruijm · O. Bonny · M. Burnier · A. Zanchi

Service of Nephrology and Hypertension, Department of Medicine, Centre Hospitalier Universitaire Vaudois, Lausanne, Switzerland

\section{A. Zanchi ( $\square)$}

Service of Endocrinology, Diabetes and Metabolism, Centre Hospitalier Universitaire Vaudois, Lausanne, Switzerland

e-mail: Anne.Zanchi@chuv.ch and chronic renal effects of empagliflozin $10 \mathrm{mg}$. The primary outcome is the effects of empagliflozin on renal tissue oxygenation as measured by BOLD-MRI. The secondary outcomes include the effects of empagliflozin on tubular function, $24 \mathrm{~h}$ blood pressure control, and the influence of body mass index (BMI) on the renal response to empagliflozin. Fifteen normal weight, 15 overweight, and 15 obese non-diabetic subjects (men and women) will be recruited. Each participant will undergo $24 \mathrm{~h}$ urine collections and blood pressure measurements on day -1 , followed by an investigation day at the study center with blood and urine sampling and renal BOLD-MRI measurements before and $180 \mathrm{~min}$ after the administration of $10 \mathrm{mg}$ empagliflozin or placebo. This sequence of measurements will be repeated after 1 month of a daily empagliflozin or placebo intake. To investigate renal oxygenation, the renal cortical and medullary R2*, as a marker of oxygenation, will be assessed by BOLD-MRI under standardized hydration conditions: the higher $\mathrm{R} 2{ }^{*}$, the lower oxygenation.

Conclusion: SGLT-2 inhibitors have a profound effect on renal physiology. This is an important study that will explore for the first time whether inhibiting SGLT-2 with empagliflozin in healthy volunteers affects renal tissue oxygenation as determined by BOLD-MRI.

Funding: Boehringer Ingelheim Pharma $\mathrm{GmbH}$ $\&$ Co. 
Trial registration: ClinicalTrials.gov identifier, NCT03093103.

Keywords: BOLD-MRI; Empagliflozin; Endocrinology; SGLT-2

\section{INTRODUCTION}

SGLT-2 inhibitors (SGLT-2i) belong to a new class of hypoglycemic drugs with the unique property of decreasing blood glucose independently from insulin, through an increase in glycosuria. Following the results of the EMPAREG trial [1, 2], the nephrology community has expressed a considerable interest in this new antidiabetic class [3]. Diabetic kidney disease is now the main cause of end-stage renal disease in developed countries and new therapies have been lacking for more than 15 years since the milestone trials with renin-angiotensin system blockers [4-6].

In high cardiovascular risk patients with type 2 diabetes (T2D), empagliflozin decreases cardiovascular mortality, death from any cause, and hospitalization for heart failure [1]. In addition, empagliflozin slows the eGFR (estimated glomerular filtration rate) decline, decreases the progression of albuminuria, and decreases cardiovascular mortality in albuminuric subjects [2]. Following these results from the EMPA-REG trial, the recommended pharmacological approach of people with T2D and established cardiovascular disease has been revised $[7,8]$.

The mechanisms of nephroprotection with SGLT-2i are under investigation. These drugs inhibit partially the sodium glucose transporter 2 (SGLT-2) in healthy volunteers and in T2D and shift the threshold for renal glucose excretion to lower levels [9]. SGLT-2 transporters are expressed at the luminal membrane of the proximal tubule and are involved in the reabsorption of $90 \%$ of filtered glucose [10]. Through SGLT-2, glucose is transported with sodium (1:1), driven by a concentration gradient induced by the active $\mathrm{Na} / \mathrm{K}$ ATPase transport of sodium at the basolateral membrane. Overall these glucose and sodium transports participate in the high metabolic activity of the proximal tubule; therefore, interacting with them may change the oxygen requirements.

The current main hypotheses for the mechanisms of nephroprotection with SGLT-2i are (1) decreased hyperfiltration due to a restored tubular glomerular feedback; (2) shift towards more favorable renal fuel energetics with improved renal oxygenation [11-14]. Indeed, adequate renal tissue oxygenation is critical for the maintenance of normal renal function [15] and hypoxia increases the susceptibility to acute and chronic renal injury [16]. Renal tissue hypoxia can be induced by oxidative stress, altered renal hemodynamics, increased GFR, tubular hypertrophy, or increased transport activity, factors that are all possibly encountered in diabetes. Type 2 diabetes is associated with lower medullary $p \mathrm{O}_{2}$ levels (measured with blood oxygenation level-dependent magnetic resonance imaging, BOLD-MRI) as compared to age-matched healthy controls [17-19]. Previous studies in humans have strongly suggested that tubular sodium reabsorption is the most important factor determining renal tissue oxygenation. Thus a reduction in proximal sodium reabsorption induced by inhibition of proximal SGLT-2 could also be associated with a favorable change in renal tissue oxygenation.

Since directly quantifying intrarenal blood flow or oxygenation is not possible in clinical practice, the detection of renal hypoxia remains indirect [15]. New forms of technology such as BOLD-MRI allow one to assess renal tissue oxygenation non-invasively [18]. The measurements can be repeated several times in the same person without the need to administer contrast products, making BOLD-MRI an ideal tool to measure medication-induced changes in renal oxygenation. This technique has been used successfully in the Nephrology Department of the University Hospital in Lausanne for more than 5 years, as illustrated by several publications [19-21]. We have shown previously that a decrease in sodium intake in healthy subjects and hypertensive patients increases renal tissue oxygenation [20]. Similarly, the administration of furosemide has been associated with a decrease in sodium reabsorption and an increase in renal tissue oxygenation. 
With this study, we propose to explore the effects of empagliflozin on renal tissue oxygenation. Our hypothesis is that SGLT-2 inhibition decreases renal cortical energy requirements with consequently an increase in renal tissue oxygenation. In this first study we chose not to investigate people with diabetes to avoid the confounding factor of blood glucose fluctuations on the BOLD-MRI signal [19]. Furthermore different weight groups will be examined for the assessment of the influence of BMI (body mass index) and baseline GFR on the renal response to SGLT-2i.

SGLT-2 inhibition does not induce hypoglycemia unless it is given with insulin or an insulin secretagogue. The main side effects are associated with the osmotic diuresis/glycosuria, i.e., increased rate of genital infection and increased risk of hypovolemia. The risk of urinary tract infection is only marginally increased. Recently rare cases $(<0.1 \%)$ of euglycemic ketoacidosis have been reported mainly in insulin-treated subjects undergoing surgery and fasting [22]. Thus, subjects taking these drugs need to be informed of the side effects, their symptoms, treatment, and preventive measures

\section{Primary Objective}

To investigate the acute and chronic effects of SGLT-2 inhibition with empagliflozin on renal tissue oxygenation as measured by BOLD-MRI.

\section{Secondary Objectives}

To evaluate the effects of empagliflozin after 4 weeks treatment on:

1. Diurnal and nocturnal glucose, sodium, potassium, uric acid, calcium, phosphate, and lithium clearances

2. Erythropoietin and hematocrit

3. Renal resistance indexes and renal volume

4. $24 \mathrm{~h}$ blood pressure measurements, diurnal and nocturnal blood pressure and pulse pressure

5. The effect of BMI on renal reponses to empagliflozin

\section{METHODS}

\section{Overall Design, Investigational Plan, and Study Population}

This study is a double-blind, randomized, placebo-controlled study that will examine per protocol the acute and chronic renal effects of empagliflozin $10 \mathrm{mg}$ in healthy non-medicated volunteers.

Fifteen normal weight (BMI $18.5-24.9 \mathrm{~kg} /$ $\mathrm{m}^{2}$ ), 15 overweight (BMI $25-29.9 \mathrm{~kg} / \mathrm{m}^{2}$ ), and 15 obese $\left(\mathrm{BMI} \geq 30 \mathrm{~kg} / \mathrm{m}^{2}\right)$ non-diabetic subjects (men and women) will be recruited with the following criteria: age 18-50 years, HbA1c $<6.5 \%$, plasma fasting glucose $<7.0 \mathrm{mmol} / \mathrm{l}$, plasma glucose level at 2 hours of oral glucose tolerance test (OGTT) $<$ $11.1 \mathrm{mmol} / \mathrm{l}$, eGFR $>60 \mathrm{ml} / \mathrm{min}$ (estimated by the creatinine-based CKD-EPI equation), urine albumin/creatinine ratio $<3.3 \mathrm{mg} / \mathrm{mmol}$, normal ultrasound, normal urine dipstick, normal hematology and chemistry results. Each participant visits the study center on seven occasions, following the routine screening visit which includes a signed informed consent followed by a complete medical history, baseline physical examination, blood test, urine dipstick, $75 \mathrm{~g}$ oral glucose tolerance test (OGTT), and a renal ultrasound. In the absence of exclusion criteria, each weight group will be randomized to placebo $(n=5)$ and empagliflozin $(n=10)$. Thus, in total, 30 subjects will be randomized to empagliflozin and 15 subjects to placebo.

The study begins with a $24 \mathrm{~h}$ ambulatory blood pressure monitoring and $24 \mathrm{~h}$ urine collection. On the following morning, the volunteer stays at the study center for a day. During the first study day (acute phase), serial blood and urine collections and two renal BOLD-MRIs before and after the oral administration of empagliflozin $10 \mathrm{mg}$ will be done. At completion of the study day, the volunteer leaves the center and takes a pill of placebo or empagliflozin each day for 28-30 days (depending on the availability of research MRI resource and menstrual cycle for women). Women will be examined during the follicular phase of the 
cycle or during the first 7 days of the contraceptive pill.

The day before the intake of the study drug (placebo or empagliflozin), the volunteer will undergo the same sequence of measurements during 2 days as described above (chronic phase). The overall study design is illustrated (Fig. 1) with detailed measurements at each visit (Table 1).

The inclusion and exclusion criteria are summarized below.

\section{Inclusion Criteria}

- Normal weight (BMI $19-24.9 \mathrm{~kg} / \mathrm{m}^{2}$ ), overweight (BMI $25-30 \mathrm{~kg} / \mathrm{m}^{2}$ ), or obesity $\left(B M I>30 \mathrm{~kg} / \mathrm{m}^{2}\right)$.

- Men and women (with a negative pregnancy test) during the follicular phase of the menstrual cycle. All groups will have a balanced number of men and women.
- OGTT/HbA1C: normal or prediabetes.

- eGFR > $60 \mathrm{ml} / \mathrm{min}$, albumin/creatinine ratio $<3.3 \mathrm{mg} / \mathrm{mmol}(<30 \mathrm{mg} / \mathrm{g})$.

- Signed consent form.

- Normal renal ultrasound.

- Normal urine dipstick.

- Normal hematology and chemistry results.

\section{Exclusion Criteria}

- Chronic medication

- NSAID treatment

- Former bariatric surgery

- Age $<18$ years or $>50$ years

- At risk of dehydration or hypotension

- Past history of recurrent genital or urinary tract infections

- Contraindication for MR imaging (pacemaker or other implanted metallic device, claustrophobia)

- Pregnancy

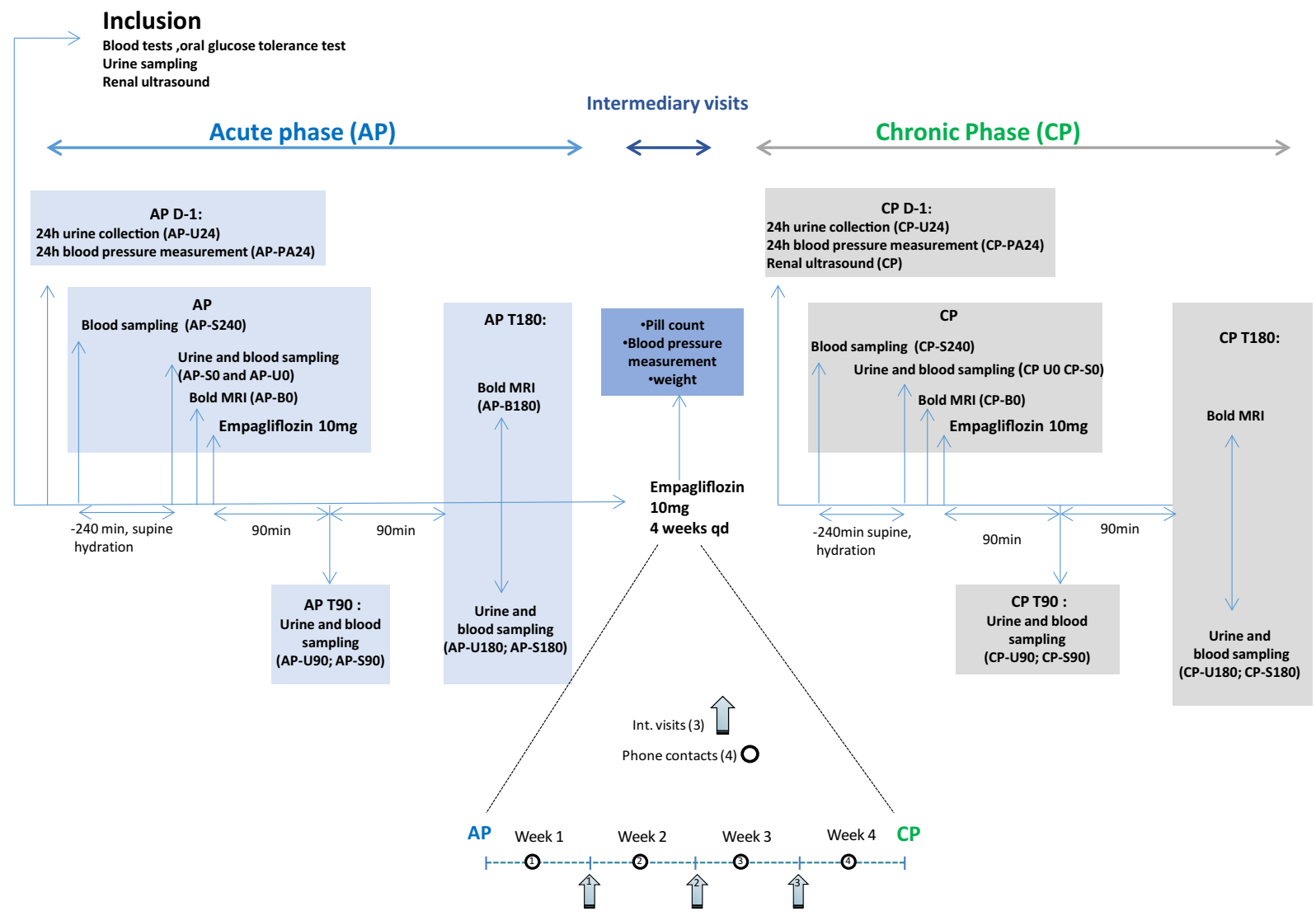

Fig. 1 Flowchart of the study 
Table 1 Detailed measurements performed at each visit

\begin{tabular}{|c|c|c|c|c|c|c|c|c|c|c|c|}
\hline & \multirow[t]{2}{*}{ Screening } & \multicolumn{5}{|c|}{ Acute phase (AP) } & \multicolumn{5}{|c|}{ Chronic phase (CP) } \\
\hline & & $\begin{array}{l}\mathrm{D}- \\
1\end{array}$ & $\begin{array}{l}\text { T- } \\
240\end{array}$ & T0 & T90 & T180 & $\begin{array}{l}\mathrm{D}- \\
1\end{array}$ & $\begin{array}{l}\text { T- } \\
240\end{array}$ & T0 & T90 & T180 \\
\hline Signed consent form & $\mathrm{X}$ & & & & & & & & & & \\
\hline Medical history & $\mathrm{X}$ & & & & & & & & & & \\
\hline Physical examination & $\mathrm{X}$ & & & & & & & & & & \\
\hline Oral glucose tolerance test & $\mathrm{X}$ & & & & & & & & & & \\
\hline Renal ultrasound & $\mathrm{X}$ & & & & & & $\mathrm{X}$ & & & & \\
\hline $\begin{array}{l}24 \mathrm{~h} \text { ambulatory blood pressure } \\
\text { measurement }\end{array}$ & & $\mathrm{X}$ & & & & & $\mathrm{X}$ & & & & \\
\hline BOLD-MRI & & & & $\mathrm{X}$ & & $\mathrm{X}$ & & & $\mathrm{X}$ & & $\mathrm{X}$ \\
\hline Pregnancy test & $\mathrm{X}$ & $\mathrm{X}$ & & & & & $\mathrm{X}$ & & & & \\
\hline Blood chemistry & $\mathrm{X}$ & & $\mathrm{X}$ & $\mathrm{X}$ & $\mathrm{X}$ & $\mathrm{X}$ & & $\mathrm{X}$ & $\mathrm{X}$ & $\mathrm{X}$ & $\mathrm{X}$ \\
\hline Blood count & $\mathrm{X}$ & & $\mathrm{X}$ & $\mathrm{X}$ & $\mathrm{X}$ & $\mathrm{X}$ & & $\mathrm{X}$ & $\mathrm{X}$ & $\mathrm{X}$ & $\mathrm{X}$ \\
\hline Urine dipstick & $\mathrm{X}$ & & & & & & & & & & \\
\hline $24 \mathrm{~h}$ urine collection & & $\mathrm{X}$ & & & & & $\mathrm{X}$ & & & & \\
\hline Urine chemistry & & & & $\mathrm{X}$ & $\mathrm{X}$ & $\mathrm{X}$ & & & $\mathrm{X}$ & $\mathrm{X}$ & $\mathrm{X}$ \\
\hline Urinary exosomes & & & & $\mathrm{X}$ & & $\mathrm{X}$ & & & $\mathrm{X}$ & & \\
\hline
\end{tabular}

- Sustained systolic blood pressure $>150 \mathrm{mmHg}$ or diastolic blood pressure $>95 \mathrm{mmHg}$

- Current or past psychiatric disease including history of substance abuse (drug, alcohol, medication)

\section{Sample Size}

The goal of this study is to examine the acute and chronic effects of empagliflozin on variations of renal tissue oxygenation, blood pressure, biochemical and clinical parameters from baseline, in comparison with placebo.

The primary goal will test the hypothesis that SGLT-2 inhibition will improve renal oxygenation with a decrease of medullary $\mathrm{R} 2{ }^{*}$, as a marker of increased oxygenation. To determine the minimum sample size needed to make adequate testing of the effect, the sample computation was based on data available from the study by Pruijm et al. [19]. The mean ( \pm SD) baseline medullary $\mathrm{R}^{*}$ in healthy subject was $29.3( \pm 2.4)$. Assuming that the treatment will improve the oxygenation by $10 \%$, the sample size required to demonstrate a significant effect with a power of $90 \%$ and an $\alpha$ type I error of $5 \%$ is 15 subjects per group. However we plan to randomize a total of 45 subjects: 15 in the placebo group and 30 subjects with empagliflozin. The power of the study to detect a significant difference between the placebo and treatment arm is then $97 \%$.

One secondary goal will be to examine the influence of body weight on the renal response to empagliflozin. As hyperfiltration increases with increased BMI, we plan to examine three groups, namely normal weight, overweight, and obese individuals. This part of the study is completely exploratory. However, assuming that the treatment will improve the 
oxygenation by $2.5 \%, 5 \%$, and $10 \%$, respectively, in the normal weight, overweight, and obese groups, sample size calculation using a two-way analysis of variance (ANOVA) with the treatment (2 levels) as a row factor and the BMI groups (3 levels) as a column factor showed that a minimal sample size of 5 subjects by cell (total $=5 \times 6=30$ ) will be needed to show the treatment effect difference between the BMI groups with a power of $80 \%$ and an $\alpha$ type I error of $5 \%$. The calculated sample size for the primary goal will then cover the secondary goal.

Because interindividual values of renal parameters may vary, we will also analyze the intraindividual variations of renal parameters compared to baseline values. We have performed similar studies in the past with serial measurements in the same subject exposed to different treatment phases [23, 24]. Although a small number of individuals were examined in these studies $(n=9-16)$, statistical significance was reached for the change in renal parameters. In addition, repeated measurements from the placebo group will examine the reproducibility of the method.

\section{Enrollment}

An announcement for recruitment is posted at the University Hospital Center (CHUV) in Lausanne, Switzerland and is visible on the web page of the institution. The study center is located in the Nephrology and Hypertension Service of the CHUV. After contacting the study nurse, volunteers receive an information sheet which includes detailed information on the study protocol, side effects, their symptoms, treatment, and preventive measures, and they are invited to an information visit. Then, a minimum of $48 \mathrm{~h}$ is requested before confirming their interest and planning the screening visit.

\section{Screening}

The screening visit begins after signing the written informed consent. Screening includes a complete medical history to confirm the participant's eligibility as determined by the inclusion and exclusion criteria, a physical examination, a blood test, a urine dipstick, an OGTT, and a renal ultrasound. A decision as to the participant's eligibility is taken as soon as all the results are available. One week to 2 months after the screening visit, the volunteer is included in the first day of the acute phase study (APJD-1) and a month later in the chronic phase study. At that time they also receive two bottles (day and night) for the $24 \mathrm{~h}$ urine collection and instructions to follow for the investigation days (APD-1, AP, CPD-1, CP) and a calendar to write down when they take the pills.

\section{Randomization}

Randomization procedure is done by the hospital's pharmacy the first day of the acute phase (APD-1). Empagliflozin $10 \mathrm{mg}$ or placebo pills are identical in size and stored in similar boxes containing 30 pills.

\section{Drug Administration}

The first pill of empagliflozin $10 \mathrm{mg}$ or placebo is administered by the study team right after the first BOLD-MRI with a glass of water. The participant is instructed to take a pill each day at the same time until the last day of the study (between day 28 and 30). The participants are asked to write down on a calendar every day at what time they take the pill and any comment they may have. At each visit, the calendar and pill count are checked to confirm drug compliance. At the end of the study, the participants return the box and its unused product that will be destroyed on-site.

\section{Study Visits and Procedures}

Volunteers undergo two examination phases: the acute phase (AP) and the chronic phase (CP).

Similar examinations are performed for the acute phase (AP: D-1, T-240, T0, T90, and T180) and the chronic phase (CP: D-1, T-240, T0, T90, T180). During the chronic phase evaluation, an additional renal ultrasound is performed as at screening. 
D - 1 (day - 1): volunteers visit the study center briefly at 8.30 a.m. to be equipped with the $24 \mathrm{~h}$ ambulatory blood pressure measurement Diasys device. Women have a pregnancy test. A renal ultrasound is performed at the chronic phase.

The rest of the day is spent routinely out of the center. Measurements include:

- A $24 \mathrm{~h}$ urine collection. Diurnal and nocturnal urine are collected separately to measure glucose, sodium, potassium, uric acid, calcium, phosphate, and endogenous lithium. Participants are instructed not to smoke or drink alcohol or have any caffeine-containing beverages during that day.

- $24 \mathrm{~h}$ ambulatory blood pressure measurements: on the day of the $24 \mathrm{~h}$ urine collection, $24 \mathrm{~h}$ ambulatory blood pressure is recorded, with measurements performed at 20-min intervals from 8 a.m. to 10 p.m., and at 30-min intervals from 10 p.m. to 8 a.m. (Diasys, Physicor, Geneva, Switzerland). This device has been validated by the British Hypertension Society and was rated B/B.

- Renal ultrasound (only at screening and chronic phase evaluation). Renal ultrasounds are performed according to a standard procedure [25] for the screening of renal abnormalities at inclusion and for the measurements of renal resistance indexes. The Aplio XG device (Toshiba Medical Systems, Volketswik, Switzerland) will be used for ultrasound examination and performed by the same experienced operator in the study center.

- For women only: a plasma pregnancy test. $\mathrm{AP} / \mathrm{CP}$ following $\mathrm{D}-1(\mathrm{~T}-240$, T0, T90, T180):

- The following day, the participant has a light breakfast at 7 a.m. at home.

- At arrival at 9 a.m. at the study center, after urine voiding, a peripheral venous catheter will be inserted for serial blood sampling. Volunteers will be hydrated initially with $5 \mathrm{ml} / \mathrm{kg}$ of oral water followed by $3 \mathrm{ml} / \mathrm{kg} / \mathrm{h}$. An identical oral hydration protocol is used for each participant on the day of each BOLD-MRI measurement to avoid as much as possible differences in renal perfusion induced by differences in volume status.
- The volunteer remains until 5 p.m. in the research center to undergo two BOLD-MRIs, urine and blood sampling. Peak levels of empagliflozin are reached $1.5-3 \mathrm{~h}$ after a single dose [26]. Each volunteer will thus undergo renal BOLD-MRI measurements before (T0) and $180 \mathrm{~min}$ (T180) after the first pill of empagliflozin $10 \mathrm{mg} /$ placebo. Urine and blood sampling will be performed at T-240, T0, T90, and T180. BOLD-MRI measurements of the kidneys will be performed at T0 and T180 at the Department of Radiology at the University Hospital CHUV, Lausanne. The research nurse escorts the participant to the BOLD-MRI and stays with him during the whole procedure which lasts around $30 \mathrm{~min}$.

All tests are repeated after 4 weeks of treatment with empagliflozin (chronic phase).

During the 4 weeks of treatment, volunteers have weekly telephone contacts with the research nurse in order to monitor the course of the treatment and identify side effects. Weekly intermediary visits are scheduled for blood pressure, weight measurements, and pill counts.

The last pill will be taken right after the $\mathrm{CP}$ T0 renal BOLD-MRI measurement.

\section{Pharmacovigilance}

All adverse events are reported and graded by the co-investigator physician in charge of the participant. All non-serious adverse events (AE) are recorded at each visit. Each volunteer carries a volunteer card indicating his randomization number, the name and number of the study, the name of the principal investigator, the name of the drug (empagliflozin or placebo), and the emergency phone number.

In case of serious adverse event (SAE), the volunteer will call the emergency number on the card and reach the nephrologist on duty. The nephrologist will decide if emergency management and suspending the treatment are necessary and inform the co-investigator in charge of the study as soon as possible. The coinvestigator will evaluate the necessity of breaking the code. If breaking the code is necessary, the co-investigator will immediately 
inform the principal investigator who keeps the randomization code in one envelope per volunteer.

\section{Trial Monitoring}

Monitoring is overseen by the clinical trial unit (CTU) according to a risk-adapted monitoring strategy described in a study-specific monitoring plan and according to standard operating procedures of the Lausanne CTU. Basically, a site initiation visit, routine monitoring visits, and a close-out visit will take place. The CTU's role includes checking that all approvals are in place before the start of the trial, the quality, security, and observation of data protection law, ensuring the trial is conducted in accordance with the International Conference on Harmonisation (ICH) Good Clinical Practice (GCP), and controlling of the adherence to the study protocol.

\section{Analysis}

Data analysis will be performed using Stata Software (StataCorp. 2015. Stata Statistical Software: Release 14. College Station, TX: StataCorp LP). Summary data will be presented as mean $( \pm \mathrm{SD})$ for continuous variables and as numbers (percent) for categorical variables. To investigate the acute and chronic effects of the SGLT-2 inhibition on the improvement of renal oxygenation, the renal cortical and medullary $\mathrm{R} 2^{*}$, as a marker of oxygenation will be assessed using a mixed-effect linear model. The variations of parameters during the study and the influence of clinical cofactors (age, sex, BMI, etc.) will be tested as well as the interactions between the BMI groups and treatment and phase. A level of significance less than 0.05 will be used in this study. An analysis of sensitivity to missing values will be performed. First, an intent-to-treat analysis including all patients will be performed. Secondly we will run multiple imputations using a Markov chain Monte Carlo single chain method based on patient baseline characteristics to create $M$ imputed data sets. Analysis will be performed separately on each imputation $m=1, \ldots, M$ and the results obtained from the $M$ completed data analyses will be combined into a single multiple-imputation result which will be compared to results from no imputed data.

Data collected in the trial will be analyzed and submitted for publication as soon as possible. Data analysis will be exclusively performed by the authors of the study with their free interpretation.

\section{BOLD-MRI Analysis}

MR images are acquired using one coronal slice on a 3-T whole-body MR system (Magnetom Prisma, Siemens Medical Systems, Erlangen, Germany), using previously described MR parameters (Pruijm, Plos ONE 2014). In brief, 12 T2*-weighted images are collected for the coronal slice within a single breath-hold of $12.4 \mathrm{~s}$ (in expiration) using a modified multi echo data image combination sequence (MEDIC) for BOLD analysis. This procedure is repeated 10 times.

All data are saved on CDs and stored in a secured place at the Nephrology and Hypertension Department of the study center. Images will be imported for further analysis in Matlab 7.11 (The MathWorks Inc., Natick, MA, USA) [27]. R2* maps will be calculated voxel by voxel using the linear least-squares fit of the logarithm of the signal. The 12-layer concentric objects method (TLCO) will be used to analyze the images. With this technique, an automatic algorithm divides the selected renal parenchyma of each coronal slice into 12 layers of equal thickness. By averaging the R2* values per layer of all four coronal slices, one obtains the mean R2* of each layer for the kidney. The mean $\mathrm{R} 2 *$ value of the outer three layers of the two kidneys will be used as a proxy of renal cortical oxygenation, and the mean $\mathrm{R} 2^{*}$ value of the inner (8th-10th) layers will be used to report medullary oxygenation. Renal blood flow in the main renal arteries will be measured using phase contrast MRI. 


\section{Study Strengths and Limitations}

- This is the first study to examine the acute and chronic effects of empagliflozin on renal oxygenation using BOLD-MRI.

- The randomized, placebo-controlled design will clearly examine the hypothesis that empagliflozin may influence renal oxygenation.

- The measurement of important variables such as $24 \mathrm{~h}$ blood pressure control, tubular function, and BMI will examine their influence on the renal responses to empagliflozin.

- The fact that non-diabetic individuals are examined may decrease the power to identify a renal effect of empagliflozin as glycosuria with SGLT-2i is less pronounced than in type 2 diabetes.

- BOLD-MRI measurements provide an indirect quantification of renal oxygenation.

\section{Ethics and Dissemination}

The research project is carried out in accordance with the research plan and the principles of the current version of the Declaration of Helsinki (DoH), the Essentials of Good Epidemiological Practice issued by Public Health Schweiz (EGEP), Swiss law, and Swiss regulatory authority requirements as applicable. The local ethics committee (EC) and regulatory authorities are informed about the project start and termination. The protocol was accepted by the local ethical committee (Commission cantonale d'éthique de la recherche sur l'être humain, protocol number CER-VD 2016-00099) and SwissMedics (no. 2016DR2171), the national drug regulation authority.

The participant receives written and oral information from the co-investigator physician before the screening visit. The participant has at least $48 \mathrm{~h}$ to think before planning the screening visit, if he is still interested in the study. He is invited to ask all questions that may arise before or at the screening visit. Then he is asked to sign the informed consent document. A copy of the signed document is given to the patient and the original placed in the investigator site file. The participant may withdraw from the trial at any time by revoking his consent without any reason.

Data collected in the trial will be analyzed and submitted for publication as soon as possible. Data analysis will be exclusively performed by the authors of the study with their free interpretation.

\section{CONCLUSION}

SGLT-2 inhibitors have a profound effect on renal physiology with nephroprotective effects in patients with T2D and established macrovascular disease. Different hypotheses on the mechanisms of nephroprotection are currently under investigation. A favorable effect on renal oxygenation has been proposed as a contributing factor. This randomized controlled study will examine for the first time whether acute or chronic inhibition of SGLT-2 with empagliflozin in healthy volunteers of different weight categories affects renal tissue oxygenation as determined by BOLD-MRI. The results of this study should lay the foundation for future studies exploring the effects of SGLT-2 inhibition on renal oxygenation in a population at risk from nephropathy using BOLD-MRI.

\section{ACKNOWLEDGEMENTS}

Funding. This study has received financial support from Boehringer Ingelheim. This study as well as the article processing charges were supported by Boehringer Ingelheim Pharma GmbH \& Co grant number 1245.127//IIS-2-028CH_Burnier. Data analysis and manuscript preparation is done independently and exclusively by participating authors. All authors had full access to all of the data in this study and take complete responsibility for the integrity of the data and accuracy of the data analysis.

Editorial Assistance. We thank Emmanuelle Dubout for assistance in preparing the protocol for Swissmedic. 
Authorship. All named authors meet the International Committee of Medical Journal Editors (ICMJE) criteria for authorship for this article, take responsibility for the integrity of the work as a whole, and have given their approval for this version to be published.

Authorship Contributions. MB is the principal investigator. AZ wrote the study protocol and is co-investigator and the corresponding author. MEM is responsible for the running of the clinical trial and is co-investigator. MEM drafted the protocol in the journal format. MP and $\mathrm{OB}$ are co-investigators. All authors have contributed to the revision of the manuscript.

Disclosures. Anne Zanchi has received funding from Boehringer Ingelheim for this study and has no other conflict of interests related to the study. Michel Burnier has received funding from Boehringer Ingelheim for this study and has no other conflict of interests related to the study. Menno Pruijm is supported by a grant from the Swiss National Science Foundation (FN320030-169191). Olivier Bonny, and Marie-Eve Muller have nothing to disclose related to this study.

Compliance with Ethics Guidelines. The research project is carried out in accordance to the research plan and with the principles of the current version of the Declaration of Helsinki (DoH), the Essentials of Good Epidemiological Practice issued by Public Health Schweiz (EGEP), Swiss law, and Swiss regulatory authority requirements as applicable. The local ethics committee (EC) and regulatory authorities are informed about the project start and termination. The protocol was accepted by the local ethical committee (Commission cantonale d'éthique de la recherché sur l'être humain, protocol number CER-VD 2016-00099) and SwissMedics (No. 2016DR2171), the national drug regulation authority. The participant receives written and oral information from the co-investigator physician before the screening visit. The participant has at least $48 \mathrm{~h}$ to think before planning the screening visit, if he is still interested in the study. He is invited to ask all questions that may arise before or at the screening visit. Then he is asked to sign the informed consent document. A copy of the signed document is given to the patient and the original placed in the investigator site file. The participant may withdraw from the trial at any time by revoking his consent without any reason.

Data Availability. The data sets used and/or analyzed during the current study will be available from the corresponding author on reasonable request once the study is completed and published.

Open Access. This article is distributed under the terms of the Creative Commons Attribution-NonCommercial 4.0 International License (http://creativecommons.org/licenses/ by-nc/4.0/), which permits any noncommercial use, distribution, and reproduction in any medium, provided you give appropriate credit to the original author(s) and the source, provide a link to the Creative Commons license, and indicate if changes were made.

\section{REFERENCES}

1. Zinman B, Wanner C, Lachin JM, et al. Empagliflozin, cardiovascular outcomes, and mortality in type 2 diabetes. N Engl J Med. 2015;373(22):2117-28.

2. Wanner C, Inzucchi SE, Zinman B. Empagliflozin and progression of kidney disease in type 2 diabetes. N Engl J Med. 2016;375(18):1801-2.

3. van Bommel EJ, Muskiet MH, Tonneijck L, Kramer $\mathrm{MH}$, Nieuwdorp $\mathrm{M}$, van Raalte DH. SGLT2 inhibition in the diabetic kidney-from mechanisms to clinical outcome. Clin J Am Soc Nephrol. 2017;12(4):700-10.

4. Lewis EJ, Hunsicker LG, Bain RP, Rohde RD. The effect of angiotensin-converting-enzyme inhibition on diabetic nephropathy. The Collaborative Study Group. N Engl J Med. 1993;329(20):1456-62.

5. Brenner BM, Cooper ME, de Zeeuw D, et al. Effects of losartan on renal and cardiovascular outcomes in patients with type 2 diabetes and nephropathy. N Engl J Med. 2001;345(12):861-9. 
6. Lewis EJ, Hunsicker LG, Clarke WR, et al. Renoprotective effect of the angiotensin-receptor antagonist irbesartan in patients with nephropathy due to type 2 diabetes. $\mathrm{N}$ Engl J Med. 2001;345(12):851-60.

7. ADA. Standards of medical care in diabetes-2017: summary of revisions. Diabetes Care. 2017;40(Suppl 1):S4-5.

8. Piepoli MF, Hoes AW, Agewall S, et al. 2016 European guidelines on cardiovascular disease prevention in clinical practice: the Sixth Joint Task Force of the European Society of Cardiology and Other Societies on Cardiovascular Disease Prevention in Clinical Practice (constituted by representatives of 10 societies and by invited experts) developed with the special contribution of the European Association for Cardiovascular Prevention \& Rehabilitation (EACPR). Eur Heart J. 2016;37(29):2315-81.

9. DeFronzo RA, Hompesch M, Kasichayanula S, et al. Characterization of renal glucose reabsorption in response to dapagliflozin in healthy subjects and subjects with type 2 diabetes. Diabetes Care. 2013;36(10):3169-76.

10. Riser Taylor S, Harris KB. The clinical efficacy and safety of sodium glucose cotransporter-2 inhibitors in adults with type 2 diabetes mellitus. Pharmacotherapy. 2013;33(9):984-99.

11. Cherney DZ, Perkins BA, Soleymanlou N, et al. Renal hemodynamic effect of sodium-glucose cotransporter 2 inhibition in patients with type 1 diabetes mellitus. Circulation. 2014;129(5):587-97.

12. Vallon V. Tubuloglomerular feedback and the control of glomerular filtration rate. News Physiol Sci. 2003;18:169-74.

13. Fioretto P, Zambon A, Rossato M, Busetto L, Vettor R. SGLT2 inhibitors and the diabetic kidney. Diabetes Care. 2016;39(Suppl 2):S165-71.

14. Mudaliar S, Alloju S, Henry RR. Can a shift in fuel energetics explain the beneficial cardiorenal outcomes in the EMPA-REG OUTCOME study? A unifying hypothesis. Diabetes Care. 2016;39(7):1115-22.

15. Brezis M, Rosen S. Hypoxia of the renal medullaits implications for disease. $\mathrm{N}$ Engl J Med. 1995;332(10):647-55.

16. Thadhani R, Pascual M, Bonventre JV. Acute renal failure. N Engl J Med. 1996;334(22):1448-60.

17. Yin WJ, Liu F, Li XM, et al. Noninvasive evaluation of renal oxygenation in diabetic nephropathy by BOLD-MRI. Eur J Radiol. 2012;81(7):1426-31.
18. Prasad PV. Evaluation of intra-renal oxygenation by BOLD MRI. Nephron Clin Pract. 2006;103(2):c58-65.

19. Pruijm M, Hofmann L, Piskunowicz $M$, et al. Determinants of renal tissue oxygenation as measured with BOLD-MRI in chronic kidney disease and hypertension in humans. PLoS One. 2014;9(4):e95895.

20. Pruijm M, Hofmann L, Maillard M, et al. Effect of sodium loading/depletion on renal oxygenation in young normotensive and hypertensive men. Hypertension. 2010;55(5):1116-22.

21. Pruijm M, Hofmann L, Charollais-Thoenig J, et al. Effect of dark chocolate on renal tissue oxygenation as measured by BOLD-MRI in healthy volunteers. Clin Nephrol. 2013;80(3):211-7.

22. Peters AL, Buschur EO, Buse JB, Cohan P, Diner JC, Hirsch IB. Euglycemic diabetic ketoacidosis: a potential complication of treatment with sodiumglucose cotransporter 2 inhibition. Diabetes Care. 2015;38(9):1687-93.

23. Zanchi A, Maillard M, Jornayvaz FR, et al. Effects of the peroxisome proliferator-activated receptor (PPAR)-gamma agonist pioglitazone on renal and hormonal responses to salt in diabetic and hypertensive individuals. Diabetologia. 2010;53(8):1568-75.

24. Zanchi A, Chiolero A, Maillard M, Nussberger J, Brunner HR, Burnier M. Effects of the peroxisomal proliferator-activated receptor-gamma agonist pioglitazone on renal and hormonal responses to salt in healthy men. J Clin Endocrinol Metab. 2004;89(3):1140-5.

25. Pruijm M, Ponte B, Ackermann D, et al. Heritability, determinants and reference values of renal length: a family-based population study. Eur Radiol. 2013;23(10):2899-905.

26. Heise T, Seman L, Macha S, Jones P, Marquart A, Pinnetti S, et al. Safety, tolerability, pharmacokinetics, and pharmacodynamics of multiple rising doses of empagliflozin in patients with type 2 diabetes mellitus. Diabetes Ther. 2013;4(2):331-45.

27. Milani B, Ansaloni A, Sousa-Guimaraes S, et al. Reduction of cortical oxygenation in chronic kidney disease: evidence obtained with a new analysis method of blood oxygenation level-dependent magnetic resonance imaging. Nephrol Dial Transplant. 2017;32(12):2097-105. 